\title{
Optimizing Radiologic Workup: An Artificial Intelligence Approach
}

\author{
Henry A. Swett, Michael Rothschild, Gregory G. Weltin, Paul R. Fisher, and Perry L. Miller
}

The increasing complexity of diagnostic imaging is presenting an ever expanding variety of radiologic test options to clinicians. As a result, it is becoming more difficult for referring physicians to select an appropriate sequence of tests. The current economic pressures on medicine make it particularly important that resources be used judiciously. Radiologic workup often involves a sequence of tests that lead from presenting signs and symptoms to a definitive diagnosis or intervention. This sequence ideally begins with simple, inexpensive, safe, non-invasive tests and progresses to more complex, expensive, and hazardous tests only if the simpler tests are insufficient to establish a diagnosis. DXCON is a developmental artificial intelligence-based computer system that gives advice to physicians about the optimum sequencing of radiologic tests. DxCON ovaluates basic clinical information and a physician's proposed workup plan. The system then creates an analysis of the strengths and weaknesses of his plan. The domain chosen to explore computer-based workup advice is the radiologic workup of obstructive jaundice.

- 1989 by W.B. Saunders Company

KEY WORDS: Computers, artificial intelligence, radiologic workup, obstructive jaundice.

$\mathbf{R}^{\wedge}$ APID advances in diagnostic imaging are presenting a steadily increasing variety of imaging choices to referring clinicians. In the evaluation of hepatobiliary disease, for example, there are at least ten different ways to image the liver and biliary tract ranging from simple plain films, through computed tomography (CT) and ultrasound (US) to invasive modalities such as PTC and ERCP. It makes good medical sense to use these modalities appropriately and in proper sequence since some are complimentary while others are redundant. It is essential to reserve invasive and potentially hazardous examinations for those diagnostic problems that cannot be resolved with simpler methods. Because of uncertainty about the appropriate use of these tests and a desire to avoid workup delays, many clinicians order more examinations than necessary. Once set in motion, the imaging workup may even continue beyond the point of establishing a diagnosis.

Inappropriate workup is not only medically unsound, but is becoming economically untena- ble. Existing and proposed prospective reimbursement systems are penalizing institutions and may penalize radiologists for unnecessarily costly workup. As a result, it is critically important that radiologists assume a more active role as the architects of imaging workup plans.

Computers are a natural tool to help physicians plan workup. They are being used with increasing frequency to manage information in radiology departments and hospitals as well as display and distribute images. Artificial intelligence (AI) is an approach to the design of computer programs that allows them to apply "expert knowledge" to specific problems and thus give advice or make decisions. This report describes DxCON, a developmental AI-based computer system designed to help the physician optimize the radiologic workup of obstructive jaundice.

\section{SYSTEM FUNCTION}

\section{Domain}

The medical domain chosen to explore computer-based workup advice is the imaging workup of obstructive jaundice. For the purposes of this project, we assume that the clinician has ruled out medical causes of jaundice and wishes to evaluate obstructive etiologies. The system is designed to give advice about the appropriate use of hepatobiliary US, CT, HIDA scan, transhepatic cholangiography, and endoscopic retrograde cholangiopancreatography. The etiologies of obstructive jaundice and the workup modalities considered by DxCON are summarized in Table 1.

From the Departments of Diagnostic Radiology and Anesthesiology, Yale University School of Medicine, New Haven, CT.

Supported in part by a grant from the DeCamp Foundation, and by NIH Grants ROI-LM-04336 and TM15-LM07056 from the National Library of Medicine.

Address reprint requests to Henry A. Swett, MD, Department of Diagnostic Radiology, Yale University School of Medicine, 333 Cedar St, New Haven, CT 06510.

(1) 1989 by W.B. Saunders Company

0897-1889/89/0201-0009\$03.00/0 
Table 1. DxCON Domain: Obstructive Jaundice

\begin{tabular}{ll}
\hline \multicolumn{1}{c}{ Etiologies } & \multicolumn{1}{c}{ Imaging Modalities } \\
\hline Cholelithiasis & Ultrasound \\
Benign stricture & Computed tomography \\
Malignancy & HIDA \\
Pancreas & Transhepatic cholangiography \\
Bile ducts & Endoscopic retrograde cholangiopan- \\
Gall bladder & creatography \\
Liver & \\
Lymph nodes & \\
\hline
\end{tabular}

\section{DxCON Examples}

In order to use DxCON, the physician enters relevant clinical information and data concerning prior imaging workup via a series of menu selections and then specifies a new examination which s/he proposes to evaluate the patient further. DxCON responds to this proposal by producing a detailed critique of the strengths and weaknesses of the proposed workup. This critique is tailored around the patient's specific clinical findings and the referring clinicians workup plan. In addition to DxCON's detailed discussion, a brief summary form of output is available. The workup discussions produced by DxCON attempt to explain the rationale behind appropriate test selection. The final decision concerning test selection is made by the referring physician, not the computer.

When the proposed imaging workup is supported by the programs logic, DxCON produces a discussion that summarizes both the indications for the selected test and the system's conclusion that the test is indicated in the clinical setting that has been described (Fig 1). This serves to reinforce the rationale behind a correct workup decision. When the system identifies a workup proposal that may be inappropriate, it explains why the selected test is not indicated and reviews the usual indications for that test. A preferred alternate examination is suggested if appropriate (Fig 2). The system can suggest that a diagnosis has been established with sufficient certainty that no further workup is required.

DxCON looks at several aspects of a workup in progress. It considers the appropriateness of both the examination requested and each relevant imaging study already performed. Thus the system will discuss workup errors already made for future reference while reinforcing correct workup decisions (Figs 2 and 3). When an invasive study is contemplated, DxCON summarizes the implications of the noninvasive studies, which have already been performed or should have been performed, and then considers the appropriate use of an invasive study (Fig 3).

\section{SYSTEM DESIGN}

DxCON is an expert system that uses "if-then rules" to evaluate the available clinical information. These rules establish the set of known facts about a case and are used to evaluate the workup implications of these facts. The program assembles an English language discussion of the workup plan by piecing together fragments of sentences or paragraphs into a logically flowing analysis.

More specifically, DxCON produces a block of data known as a facts list from the data
Case E14EA: A 74 year old man who presents with shaking chills, vomiting, chest pain, dark urine, light stool, and hyperbilirubinemia. Ultrasound is requested.

\section{DxCON Discussion:}

\section{NONINVASIVE STUDIES}

Ultrasound is usually the initial study in the evaluation of suspected obstructive jaundice because of its low cost, portability, and excellent sensitivity for biliary dilatation, gallstones, hepatic masses, and pancreatic pathology. We therefore agree with the choice of ultrasound as the initial study in this patient.
Fig 1. DxCON discussion which supports the proposed workup. 
Fig 2. DxCON discussion which disagrees with the planned workup.
Case E11: 74 year old woman with acute severe generalized epigastric pain and tenderness. CT was done first and was unremarkable. An ultrasound was ordered.

\section{DXCON Discussion: NONINVASIVE STUDIES}

Ultrasound is usually preferred to CT for the initial evaluation of suspected obstructive jaundice because of its low cost, portability, and excellent sensitivity for biliary dilatation, gallstones, hepatic masses, and pancreatic pathology. In a very obese patient, the US examination is frequently limited and CT is therefore preferred. Since this patient is not obese, we see no reason to perform a CT scan before doing the ultrasound examination.

Ultrasound is appropriate to confirm or exclude a suspected abnormality when the initial CT study suggests the possibility of an intraductal stone or a mass lesion but cannot resolve the question. As there was no such question and the CT study was otherwise satisfactory, we see no reason for a subsequent ultrasound.

A HIDA scan can detect the presence of biliary obstruction in the absence of biliary dilatation, which can occur in up to $10 \%$ of cases. Typically, it would be included in this patient's workup.

Case E18LB: This is an 87 y/0 man with a history of cholecystectomy. He presents with acute onset of right upper quadrant pain, nausea, vomiting, fever, and jaundice. He was made NPO on admission. Ultrasound was significant only for a dilated common bile duct. HIDA scan was felt to be consistant with a partial obstruction of the CBD. An ERCP has been ordered.

\section{DxCON Discussion:}

Ultrasound is usually the initial study in the evaluation of suspected obstructive jaundice because of its low cost, portability, and excellent sensitivity for biliary dilatation, gallstones, hepatic masses, and pancreatic pathology. We therefore agree with the choice of ultrasound as the initial study in this patient.

A HIDA scan can detect the presence of biliary obstruction in the absence of biliary dilatation, which can occur in up to $10 \%$ of cases. In the presence of biliary dilatation already documented by US or CT, little if any additional information was to be expected from this examination.

\section{NONINVASIVE STUDY RESULTS}

A dilated common bile duct in the absence of an obvious cause can be due to a wide variety of lesions both benign and malignant, but suggests an obstructing lesion at the level of the distal common bile duct, ampulla of Vater, duodenum, or pancreatic head. When the patient has a history of gallstones or cholecytectomy, the possibility of a stone below the limits of imaging resolution or a benign common bile duct stricture must be seriously considerred.

\section{INVASIVE STUDIES}

Invasive procedures used in the workup of obstructive jaundice include percutaneous transhepatic cholangiography (PTC) and endoscopic retrograde cholangiopancreatography (ERCP). PTC and ERCP are in many ways complimentary procedures, and the optimal choice often depends on local expertise and experience. These are appropriate when the noninvasive tests indicate obstruction without adequately demonstrating its etiology. As this is the case, an invasive examination is indeed appropriate.

Fig 3. DxCON discussion of a complex case. 
entered by the physician. The items on this list are primary clinical facts, that is, they were explicitly stated by the physician to be true and are dependent on no other conditions. The system next applies a set of if-then rules to the given facts. This set of rules generates additional facts which have been inferred from the primary facts. A set of expressive frames evaluate the given and inferred facts and organize the general flow of the discussion based on general jaundice workup principles. The expressive frames point to a series of comment frames contained in a prose network which contain the actural comments that will be used in the final critique. These comments are logically concatanated to make up a natural sounding English language discussion. ${ }^{1}$

To understand this better, consider the second paragraph of the discussion in Fig 3: "A HIDA scan can detect the presence of biliary obstruction in the absence of biliary dilatation, which can occur in up to $10 \%$ of cases. In the presence of biliary dilatation already documented by US or CT, little if any additional information was to be expected from this examination." The relevant facts that were given include: (1) Ultrasound showed a dilated common bile duct, and (2) HIDA scan consistent with partial obstruction of the common bile duct (CBD).

A partial list of inferred facts (from "if-then" rules) includes: (1) ultrasound was done, (2) ultrasound was satisfactory, (3) HIDA was done, (4) HIDA was satisfactory, (5) an initial imaging study was done, (6) a potentially obstructing lesion of CBD was demonstrated, (7) CBD dilated on US, and (8) CBD dilated on HIDA.

The following expressive frames (among others), in paraphrase, indicate prose fragments to be included: if an ultrasound was done before an HIDA scan and the CBD was dilated on US, THEN include HIDA comment, sequence 3. This then refers to a prose network which is evaluated as follows: HIDA INTRO-An HIDA scan can detect the presence of biliary obstruction in the absence of biliary dilatation COMMA which can occur in up to $\mid 10 \%$ of $\mid$ cases *PERIOD \$POPTT T (return to sequencer) (Imbedded expressions are operators that govern such variables as test options, punctuation, and instructions for traversing the augmented transition network expressed in the syn- tax of the Lisp computer language. ${ }^{1}$ HIDA sequence 3-In the presence of biliary dilatation already documented by US or CT ${ }^{*}$ COMMA little if any additional information ( ${ }^{*} \mathrm{CHOICE}$ ((SAME DONE HIDA YES) (was)))(T(is))) to be expected from this examination *PERIOD) SPOPTT T (return to sequencer. ${ }^{1,2}$ Higher level logic assembles these paragraphs into the final critique.

DxCON uses other domain independent programming tools to handle specific aspects of case processing and system development including Essential-Attending ${ }^{2}$ and Hydra. ${ }^{3}$ A "knowledge exerciser" was also developed to make it possible for the system developer to test how the system reacts to varying clinical circumstances. ${ }^{4}$ DxCON and all related programs are written in the LISP programming language and have been implemented on VAX (Digital Equipment Corporation, Maynard, MA) and Macintosh (Apple Computer, Inc., Cupertino, CA) computers.

\section{DISCUSSION}

Diagnostic imaging alternatives are growing at an unprecedented rate due to the development of new imaging modalities and the more sophisticated use of existing techniques. While this has enabled radiologists to play a more critical role in patient management, it has greatly complicated the process of selecting appropriate examinations. What are the best screening examinations? Which are most specific, sensitive, and accurate? How can considerations such as cost effectiveness, optimal medical practice, patient morbidity, and yield be balanced? It is sometimes difficult for radiologists to integrate these factors, and often impossible for referring physicians. The result may be that examinations are performed that are not indicated and are misleading, redundant, or unnecessarily invasive. Some clinicians resort to a "shot gun" approach to workup. This is not only intellectually unsatisfactory and potentially hazardous to patients, but is becoming economically untenable. Strong new incentives exist, or are being created, that place a fresh sense of urgency on the optimization of imaging workup.

Computers are proliferating in hospitals, radiology departments, and physician offices. These 
not only manage the financial and administrative components of medical care, but are increasingly used for clinical purposes. In a growing number of centers, orders for diagnostic imaging procedures are issued from computer terminals in patient care areas and are transmitted directly to computers in the radiology department which automatically schedule examinations, inform the floor of the patient appointments, and ultimately of the results of the examination. This electronic environment provides an opportunity for computers to not only exchange information, but to participate actively in the process of workup design. While there are a variety of ways that the computer might do this, we have been interested in the use of computer-based artificial intelligence methods for giving advice to physicians.

AI computer programs attempt to give advice or make decisions based on applying "expert knowledge" of highly specific problem domains. Medical expert systems are AI programs that often use rules or domain specific principles to help physicians solve clinical problems. We have developed several such expert systems which have explored methods for giving advice about medical management and differential diagnosis. ${ }^{5-8}$ A central design philosophy in these programs has been that the computer must not only be able to give advice, but must also be able to explain the rationale behind its advice in a way that allows the physician to determine whether the advice makes sense.

We have explored the suitability of using an expert system to give advice to referring physicians about optimizing radiologic test selection in an experimental AI program known as DxCON. DxCON does not tell the physician what to do, but rather asks about the patient and about the workup plan that the physician thinks is appropriate. DxCON then discusses the strengths and weaknesses of the the physician's plan in an English prose discussion which is structured around his or her thinking. Importantly, DxCON explains its conclusions in a way that allows the physician to evaluate the appropriateness of its advice for the specific patient. It is up to the physician to make the final decision as to workup design.

Algorithms or decision trees may be useful guides to test selection and have received re- newed attention in recent years. DxCON contains a general algorithmic framework but has the added flexibility of automatically applying that framework to a specfic clinical case. This makes it possible to rapidly identify potentially flawed workup plans and to explain the reasons why the plan appears to be inappropriate. Some workup algorithms have been criticized because they are inflexible and enforce a single workup philosophy. An added advantage of DxCON's explanatory capability is that the program can accommodate subtle differences in individual patients, local practice variations, differences in physician experience, and available facilities. The ability of our program to consider the pros and cons of a particular approach adds flexibility, but DxCON does express a specific point of view concerning the appropriate workup of obstructive jaundice, just as human experts do when providing consultation. We believe that these kinds of systems would be of limited usefulness if they did not take a stand. This presents a problem when experts disagree as to how to handle a specific situation. We have undertaken a separate study to estimate the prevalence of conflicting expertise locally. ${ }^{8}$ We have also developed an experimental system that is able to present more than one perspective when there are several dominant schools of thought. ${ }^{9}$

We have not employed a statistical approach to these problems, although such methods can be very valuable when meaningful data is available. Greenes has emphasized the importance of considering a priori and a posteriori (Bayesian) probabilities of disease as well as specificity and sensitivity as criteria for test selection. ${ }^{10}$ When reliable statistics are not available, the more subjective approach represented by DxCON may be quite successful at dealing with the vagaries of everyday practice. As seen in Figs 2 and 3 , statistical information can be included in DxCON discussion.

A drawback of all methods currently used (including ours) is that the physician must consciously consult the computer and thus, advice is only available when the physician is aware that $\mathrm{s} /$ he needs help. It would be of no value when a physician erroneously thinks that $s /$ he understands the appropriate use of the available modalities. If incorporated into a hospital infor- 
mation system, however, the computer could silently observe the radiologic test requisitioning process and only offer its advice when it recognizes a possible violation of its expectations for appropriate workup.

An additional problem with the expert system approach is that such systems are difficult to construct using today's tools and are therefore difficult to propagate in other domains. Expert system development tools, however, may make it possible for computer illiterate clinical experts to create such systems in the future. There are significant legal and regulatory problems that arise when computers assume a more active role in medical care. The critiquing approach may minimize these problems since the computer gives explanations (like a medical textbook) that allow the physician to make informed decisions. The computer is not taking direct action or providing output in a form that cannot be readily evaluated by the physician. Finally, a great deal of work needs to be done to make computerbased advice systems easy to use. They must fit seamlessly into the environment in which they will be used without being obtrusive. Such systems may require entry of a significant amount of clinical information. Ultimately, we believe that relevant clinical information will be automatically extracted from the hospital's computer systems.

\section{REFERENCES}

1. Woods WA: Transition network grammars for natural language analysis. Commun Assoc Comput Mach 13:591606, 1970

2. Miller PL: Building an expert critiquing system: Essential Attending. Methods Inform Med 25:71-78, 1986

3. Miller PL, Blumenfrucht SJ, Rose JR, Hydra: A knowledge acquisition tool for expert systems that critique medical workup. Med Decis Making 7:12-21, 1987

4. Swett HA, Rothschild MA, Weltin GG, et al: Essential-Attendings knowledge exerciser program: A tool for knowledge verification, in Proceedings of the American Association for Medical Systems and Informatics Cong 861986 , pp 194-197

5. Miller PL: Critiquing anesthetic management: The "ATTENDING" computer system. Anesthesiology 58:362369,1983

6. Miller PL, Black HR: Medical plan-analysis by com- puter: Critiquing the pharmacologic management of essential hypertension. Comput Biomed Res 17:38-54, 1984

7. Swett HA, Miller PL: ICON: A computer-based approach to differential diagnosis in radiology. Radiology 163:555-558, 1987

8. Rothschild MA, Miller PL, Fisher PR, et al: Confronting subjective criteria in the evaluation of computer-based critiquing advice, in Proceedings of the 1988 Symposium on Computer Applications in Medical Care, Washington, DC, Institute of Electrical and Electronic Engineers Computer Society, 1988, pp 220-224

9. Miller PL, Blumenfrucht SJ, Black HR: An expert system which critiques patient workup: Modeling conflicting expertise. Comput Biomed Res 17:554-569, 1984

10. Greenes RA: Computer-aided diagnostic strategy selection. Radiol Clin North Am 24:105-120, 1986 